\title{
Where Do Solar Filaments Form?
}

\author{
Duncan H Mackay ${ }^{1}$, Victor Gaizauskas ${ }^{2}$ and Anthony R. Yeates ${ }^{3}$ \\ ${ }^{1}$ School of Mathematics and Statistics, University of St Andrews, St Andrews, KY16 9SS, UK \\ email: duncan@mcs.st-and.ac.uk \\ ${ }^{2}$ HIA, CNRC, 100 Sussex Drive, Ottawa, Ontario, Canada \\ ${ }^{3}$ Department of Mathematical Sciences, Durham University, Durham, UK
}

\begin{abstract}
In the present study, we consider where large, stable solar filaments form relative to underlying magnetic polarities. We find that $92 \%$ of all large stable filaments form in magnetic configurations involving the interaction of two or more bipoles. Only $7 \%$ form above the Polarity Inversion Line (PIL) of a single bipole. This indicates that a key element in the formation of largescale stable filaments is the convergence of magnetic flux, resulting in either flux cancellation or coronal reconnection.
\end{abstract}

Keywords. Sun: filaments, Sun: magnetic fields, Sun: prominences

\section{Introduction}

Solar filaments (prominences) form over a wide range of latitudes on the Sun. One early classification scheme splits them into two categories. Tandberg-Hanssen (1995) describes these two categories as Type A (bipolar region filament) and Type B (between bipolar region filament) respectively. Observations by Tang (1987) have shown that over $60 \%$ of all filaments form between bipolar regions (Type B; see also Gaizauskas \& Zwaan(1997)). We re-examine where large, stable solar filaments form relative to underlying magnetic polarities, extending the study of Tang (1987) to cover the full solar cycle.

\section{Classification Scheme and Data Sets}

To full categorise the magnetic interactions that lead to the formation of filaments we introduce 4 categories. These are,

(a) Interior BR Filament (IBR): forms above the PIL of a single magnetic bipole.

(b) Exterior BR Filament (EBR): forms above the PIL that lies between two or more magnetic bipoles.

(c) Interior/Exterior BR Filament (I/EBR): forms above both the internal and external PIL surrounding a bipole.

(d) Diffuse BR Filament (DBR): a filament that lies above a PIL that sits in a bipolar distribution at high latitudes, where the bipolar distribution at high latitudes was produced from earlier emergences and cancellations of flux at lower latitudes.

Four distinct periods are analysed over Solar Cycle 21 where in total 603 filaments are considered. The periods studied are: Set 1: 1977, CR1653-1658, 101 Filaments; Set 2: 1979, CR1680-1685, 234 Filaments; Set 3: 1982, CR1720-1725, 149 Filaments; Set 4: 1984, CR1747-1752, 119 Filaments. For each time period the classification of each filament is carried out through a 2 stage process: (1) Use synoptic data to determine filament locations and identify underlying magnetic flux and (2) Use high resolution data and simulations to classify each filament. 


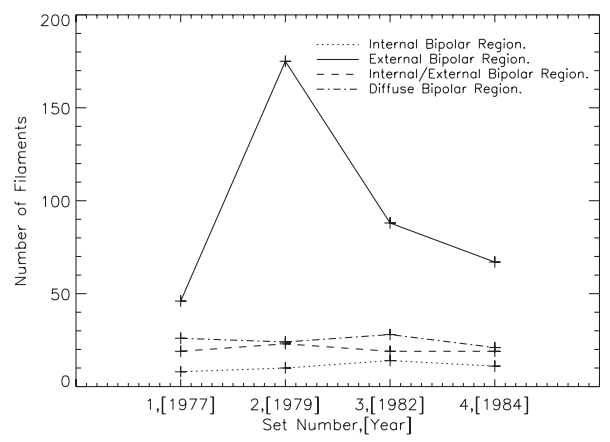

Figure 1. Number of filaments in each category as a function of time in Cycle 21.

Table 1. Percentage of filaments in each category

\begin{tabular}{c|c|c|c|c|c|c}
\hline Type & IBR & EBR & I/EBR & \multicolumn{2}{|c|}{ DBR } & $\mathbf{U}$ \\
\hline$\%$ & 7 & 63 & 13 & 16 & 1 \\
\hline
\end{tabular}

\section{Results}

Figure 1 shows the number of filaments in each category as a function of data set number. The plus signs denote the number of filaments in each category, while the varying line-style denotes the overall cycle variation of each category. Cycle maximum lies midway between Sets 2 and 3. For each of the four data sets a similar behaviour is found. EBR filaments (solid line) dominate. This graph clearly shows that filaments prefer to form in flux systems involving multiple bipole interactions rather than just a single magnetic bipole. Throughout the cycle only EBR filaments show a strong variation. This indicates that the formation mechanism for these must be closely related to the number of bipoles on the Sun throughout the solar cycle. Table 1 gives the percentage of filaments in each category calculated over the combined data sets (where "U" is unclassified). Over $92 \%$ of all filaments (EBR, I/EBR, DBR) occur in flux distributions that are non-bipolar in nature and require the interaction of two or more bipoles.

\section{Conclusions}

Over $92 \%$ of all filaments occur in flux distributions that are non-bipolar in nature and require the interaction of two or more bipoles. Since the vast majority of large-scale stable filaments occur along PILs that are external to any one bipole, we conclude that a key element in the formation of the majority of these solar filaments must be the convergence of magnetic flux between bipoles, resulting in either flux cancellation (van Ballegooijen \& Martens(1989)) or coronal reconnection (Galsgaard \& Longbottom(1999)). The consequences of this for theoretical models is discussed in Mackay et al. (2008).

\section{References}

Gaizauskas, V. \& Zwaan, C. 1997, Bulletin of the American Astronomical Society, 29, 902

Galsgaard, K. \& Longbottom, A. W. 1999, ApJ, 510, 444

Mackay, D. H., Gaizauskas, V., \& Yeates, A. R. 2008, Solar Phys., 248, 51

Tang, F. 1987, Solar Phys., 107, 233

Tandberg-Hanssen, E. 1995, Astrophysics and Space Science Library, 199,

van Ballegooijen, A. A. \& Martens, P. C. H. 1989, ApJ, 343, 971 